\title{
A Novel Electrically Tunable Long-Period Fiber Grating Using a Liquid Crystal Cladding Layer
}

\author{
A. Czapla ${ }^{a, b, *}$, T.R. Woliński ${ }^{a}$, E. Nowinowski-Kruszelnicki ${ }^{\mathrm{c}}$ And W.J. Bock ${ }^{b}$ \\ ${ }^{a}$ Faculty of Physics, Warsaw University of Technology, Koszykowa 75, 00-662 Warszawa, Poland \\ ${ }^{b}$ Photonics Reaserch Center, Univ. du Québec en Outaouais \\ Rue Saint-Jean-Bosco 101, J8X 3X7 Gatineau, Quebec, Canada \\ ${ }^{\mathrm{c}}$ Military University of Technology, Gen. S. Kaliskiego 2, 00-908 Warszawa, Poland
}

\begin{abstract}
The main aim of the presented work is the integration of a long-period fiber grating and a liquid crystal into a hybrid structure, in order to develop an innovative fiber optic device controlled by an external electric field. The studied long-period fiber grating was fabricated using UV irradiation in a boron co-doped fiber (PS1250/ 1500, manufactured by Fibercore). As a liquid crystal we used a typical 5CB nematic liquid crystal. The sensing mechanism of the proposed loss filter relies on long-period fiber grating attenuation bands sensitivity to optical properties of the liquid crystal layer. The results obtained show that the long-period fiber grating with a nanosized liquid crystal layer exhibits one order of magnitude higher electrical sensitivity and a lower level of the voltage control than the long-period fiber grating with a micro-sized liquid crystal layer.

PACS numbers: 42.81.Cn, 42.79.Dj, 42.81.Pa, 42.79.Kr
\end{abstract}

\section{Introduction}

In order to achieve light coupling between two co-propagating modes, a grating formed in an optical fiber usually requires a pitch of several hundred micrometers. This type of grating is called a long-period fiber grating (LPFG). Compared to other optical devices, LPFGs have a number of unique advantages such as low-level back reflection, low insertion losses and compact construction (the grating is an intrinsic fiber device). The LPFGs have found a variety of applications in optical communications $[1,2]$. Tuning of the LPFGs is very attractive since it can offer a form of dynamic spectral control [3, 4]. The central attenuation wavelength of the LPFGs is highly sensitive to temperature, strain, bend or surrounding refractive index (SRI) changes. For these reasons filters based on the LPFGs have generated a significant interest for applications in the sensing field as well.

Liquid crystals (LCs) are attractive candidates as a coating material since their properties can be controlled through temperature, optical, electrical and magnetic fields [5-7]. There are three key points for a good design in order to combine a LPFG and a LC into one component: selection of the LC refractive indices, inherent SRI sensitivity of the LPFG and the LC layer thickness. Majority of LCs have refractive indices that are higher than the refractive index of the silica glass. Thus, the LPFG sensitive to SRI is limited to the range where SRI is higher than the refractive index of the fiber cladding. In this case, the core modes couple to leaky modes [8] and the dependence on the resonance wavelength on the SRI is not so straightforward. So far, most of the studies have focused on the analysis of the SRI sensitivity when the

* corresponding author; e-mail: czapla@if.pw.edu.pl
LPFG cladding is surrounded by a LC medium of infinite thickness $[9,10]$. For such an infinite thickness of the high refractive index (HRI) medium, a sufficient tuning of the resonant wavelengths of the LPFG versus the SRI is very difficult. A strong improvement in the LPFGs sensitivity can be obtained by reducing the thickness of the HRI medium to nanosize [11-13]. When a nanosized HRI layer is deposited along the LPFG, a significant modification of the distribution of cladding modes occurs leading to considerable shifts of the resonance wavelengths. This effect strongly depends on both: the layer thickness and its refractive index.

In this paper we will demonstrate a loss filter with high-efficiency electrical tuning capability based on an LPFG surrounded by a nematic LC. The sensing mechanism of the proposed loss filter relies on the sensitivity of the LPFGs attenuation bands to the optical properties of the LC layer itself. The LC layer is able to modify its properties as a direct consequence of the applied external electric field. The comparative studies are presented to account for the thickness of the LC layer: micro- and nanosized. The results obtained show that a good improvement in the electrical sensitivity can be obtained for the LPFG with nanosized LC layer. In this case, we obtain an electrical sensitivity that is four times higher and a lower level voltage is used than in the case of LPFG with microsized LC layer.

\section{Principle of operation}

Transmission characteristics of an LPFG formed in a single-mode fiber can be analyzed by the coupled-mode theory [14]. The LPFGs allow the power transfer between modes of an optical fiber. This is achieved by perturbing the phase of one mode so that it matches the phase of another one: the so-called "phase matching condition". 
The phase matching condition between the fundamental mode and the forward propagating cladding mode for the LPFG is given by

$$
\beta_{\mathrm{c} 0}-\beta_{\mathrm{cl}, m}=2 \pi / \Lambda,
$$

where $\beta_{\mathrm{c} 0}$ and $\beta_{\mathrm{cl}, m}$ are the propagation constants of the forward fundamental mode and the forward cladding mode of order $m$, respectively. The resonant wavelength, $\lambda_{\text {res, } m}$, for a specific attenuation band is expressed as follows:

$$
\lambda_{\text {res }, m}=\left(n_{\mathrm{c} 0}^{\mathrm{eff}}-n_{\mathrm{cl}, m}^{\mathrm{eff}}\right) \Lambda=\delta n_{m}^{\mathrm{eff}} \Lambda,
$$

where $n_{\mathrm{c} 0}^{\mathrm{eff}}, n_{\mathrm{cl}, m}^{\mathrm{eff}}$ and $\Lambda$ stand for the effective refractive index of the core mode, the effective refractive index of the $m$-th cladding mode and the period of the LPFG. According to Eq. (2), variations in $n_{\mathrm{c} 0}^{\mathrm{eff}}, n_{\mathrm{cl}, m}^{\mathrm{eff}}$ or $\Lambda$ will shift the position of the attenuation band. Thus from a sensing perspective, the LPFGs are of interest due to the fact that the positions of their attenuation bands are sensitive to a number of external factors.

The inherent sensitivity of the LPFGs to the SRI of the LC material tuned by the external electric field is the most important property considered in this research. Depending on the boundary condition between the cladding and the surrounding medium, the cladding modes propagate in a different manner [8-13]. In air, the cladding modes experience a total internal reflection (TIR) mechanism at the interface between the cladding and air. When the refractive index of the cladding is equal to the refractive index of the surrounding medium, the cladding has an infinitely large radius, such that the cladding modes are converted into radiation modes as a result of the lack of TIR at the cladding boundary. Thus, we cannot observe any resonant wavelength effects.

A totally new mechanism comes into play as soon as the refractive index of the cladding is higher than that of the surrounding medium. In this case two scenarios should be distinguished depending on the thickness of the layer with HRI. The first scenario assumes an infinite HRI medium. The fiber cladding now becomes leaky, due to the fact that no TIR exists. As with any interface between two dielectric media, a certain amount of reflection and refraction occurs and it is the phenomenon of external reflection that is important here. Then, the Fresnel reflection coefficients dictate the proportion of light energy that is reflected. As the HRI increases, a modification of the depth of the attenuation bands is generally expected. In addition, the cladding mode experiences a noticeable shift, which can be measured by using a high-resolution optical spectrum analyzer [8]. The second scenario considers a situation where a HRI layer surrounding the LPFG has thickness of the order of nanometers. Due to the refractive-reflective regime at the cladding-layer interface, the cladding modes in a LPFG, surrounded by nanosized HRI layer, are bound within the structure comprising the core, the cladding and the layer. It has been already proven that changes in the thickness of the nanosized HRI layer led to a significant shift of the attenuation bands in the transmission spectrum of the LPFG
[11-13]. The same mechanism occurs for a HRI with the fixed layer thickness by changing its refractive index.

\section{Experimental setup and materials}

For the needs of the present work, the LPFG was fabricated by UV irradiation in a boron co-doped photosensitive fiber (PS1250/1500, manufactured by Fibercore). The PS1250/1500 fiber gives a possibility of writing the grating directly, without any hydrogenation procedure. Co-doping this fiber with boron enhances its photosensitivity, and consequently the UV exposure time required to achieve saturation of the index change is greatly decreased. A segment of PS1250/1500 fiber, with polymer coating removed, was spliced between two segments of standard communication fiber (SM28, manufactured by Corning) and placed in contact with the amplitude mask having a periodicity of $227 \mu \mathrm{m}$. The source of the UV light was provided by an excimer laser (PulseMaster GSI Lumonics) emitting at $248 \mathrm{~nm}$ wavelength. Consequently, the fiber was irradiated by a UV light and the exposure was repeated until the index modulation has reached a sufficient level to provide the desired attenuation depth in the LPFG transmission spectrum. The length of the LPFG was $4 \mathrm{~cm}$.

As an LC material we used a typical nematic 5CB LC $[6,7]$ with the ordinary, $n_{\mathrm{o}}$ and extraordinary, $n_{\mathrm{e}}$ refractive indices 1.51 and 1.72 , correspondingly (measured at $587 \mathrm{~nm}$ ). The LC cladding external to the LPFG was formed in two ways. In the first configuration the $5 \mathrm{CB}$ LC was introduced in the whole space of the LC cell due the capillary forces. In the second configuration, a thin layer of $5 \mathrm{CB}$ was initially formed on the bare LPFG. Surface tension of the LC makes it easy to coat uniformly the LPFG making the LC behave as if it had a skin.

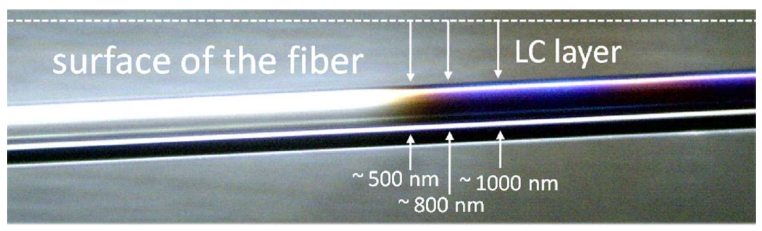

Fig. 1. Picture of the surface of the LPFG showing the border between a bare fiber and a fiber covered with a nanosize LC layer.

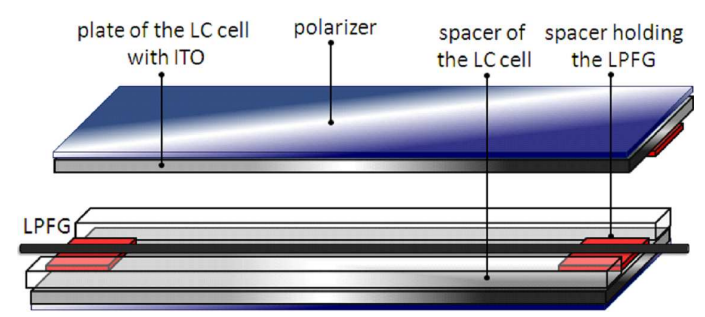

Fig. 2. Experimental setup for testing electrical sensitivity of LPFGs with LC layer. 
The presence of the LC layer on the surface of the LPFG was observed when the sample was placed between the parallel polarizers, as shown in Fig. 1. Since we know the $5 \mathrm{CB}$ birefringence $\left(0.21\right.$ at $\left.23^{\circ} \mathrm{C}\right)$, we can estimate that thickness of the LC layer is in the range from $800 \mathrm{~nm}$ to $1250 \mathrm{~nm}$ - according to the Michel-Levy interference chart $[15,16]$. Consequently, the prepared LPFG was placed in the LC cell. The construction of the $\mathrm{LC}$ cell is presented in Fig. 2. The space between the plates was $135 \mu \mathrm{m}$. In order to achieve planar orientation of the $5 \mathrm{CB}$ molecules in the LC cell, the anti-parallel rubbing was used. Indium tin oxide was deposited on inner surface of the plates to apply an electric field across the cell. Then, tuning of the LPFG was achieved by varying the boundary condition of the LC - electrically switching between $n_{\mathrm{o}}$ and $n_{\mathrm{e}}$ of the $5 \mathrm{CB}$. To achieve the optical control of the orientation of the 5CB molecules, the LC cell was operated between the crossed polarizers in such a way that they appear bright when no voltage is applied. Electrical control was conducted in the $0 \mathrm{~V} / \mu \mathrm{m}$ to $4 \mathrm{~V} / \mu \mathrm{m}$ range. The transmission spectrum was investigated with the input light projected from a halogen lamp/super continuum source and the output signal was analyzed by an Optical Spectrum Analyzer.

\section{Results and discussion}

First, the sensitivity of the LPFG to the presence of surrounding LC layer was investigated. In Fig. 2 the measured spectra of the LPFG in air, with micro- and nanosized layer of $5 \mathrm{CB}$ are presented. In the transmission spectrum of the LPFG, three main attenuation bands can be seen, designated by band 1 , band 2 and band 3 . It can be noticed that the band_ 3 is a superposition of two neighboring attenuation bands. This effect can occur if the period of the LPFG is sufficiently short $[17,18]$. When a bare LPFG is mainly surrounded by the $5 \mathrm{CB}$, a significant reduction in the depth of attenuation bands is observed and is compared in Table I. As can been seen from Fig. 3, band 3 has been strongly flattened. Therefore its measurement has become very difficult and is not presented. In addition (for both micro- and nanosized layer of $5 \mathrm{CB}$ ) the red shift of the resonant wavelengths was recorded: $2 \mathrm{~nm}$ and $1 \mathrm{~nm}$ for the band 1 and band 2, respectively.

TABLE I

comparison of the reduction of the depth of the attenuation bands due to the presence of surrounding $5 \mathrm{CB}$.

\begin{tabular}{c|c|c|c}
\hline \hline \multicolumn{3}{c}{ Reduction of the depth of the attenuation bands [\%] } \\
\hline $\begin{array}{c}\text { LPFG with infinite } \\
\text { layer 5CB }\end{array}$ & \multicolumn{2}{|c}{$\begin{array}{r}\text { LPFG with nanosized } \\
\text { layer 5CB }\end{array}$} \\
\hline Band_1 & Band_2 & Band_1 & Band_2 \\
\hline 30.26 & 62.14 & 30.26 & 62.14
\end{tabular}

In the next step we have investigated the influence of an external electric field on the spectral properties of the LPFG covered with 5 CB layers.

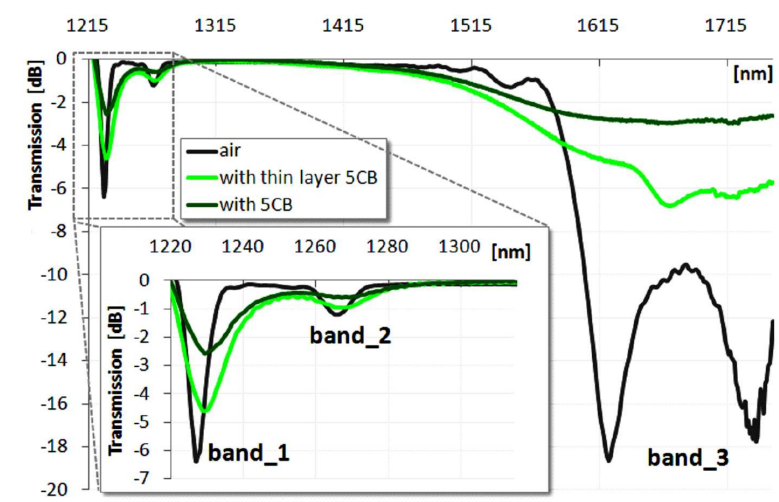

Fig. 3. Transmission spectra of the LPFG in air, with infinite and nanosized 5CB layer.

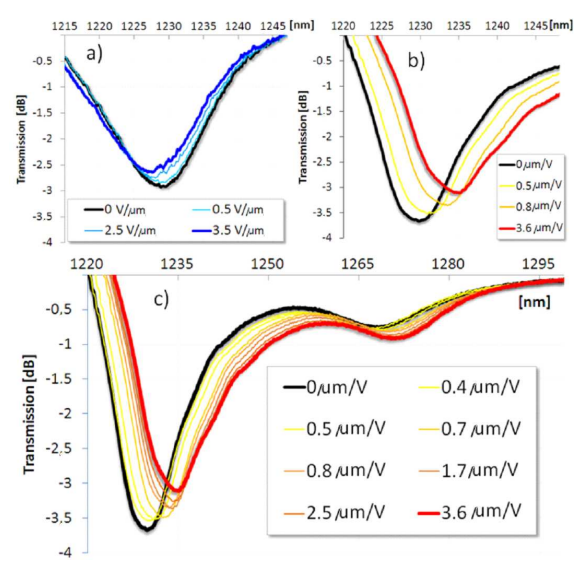

Fig. 4. Transmission spectra of LPFG with micro- (a) and nanosized (b), (c) 5CB layers.

A key fact is that optical properties of the LPFG depend strongly on the refractive index of the LC layer and consequently on orientation of the $5 \mathrm{CB}$ LC molecules. Without an electric field, an effective refractive index of the LC layer corresponds to $n_{\mathrm{o}}$ of $5 \mathrm{CB}$. Under the influence of the external electric field, a molecular reorientation occurs and at voltages higher than the threshold value, when the molecules of the LC material tend to reorient themselves perpendicularly to the fiber axis, according to the Fredericks transition rule. Thus, the refractive index of the LC layer tends to $n_{\mathrm{e}}$ of $5 \mathrm{CB}$. Consequently, the position of the attenuation bands in the transmission spectrum of the LPFG covered with the $5 \mathrm{CB}$ layer can be controlled. When the response of the LPFG with the microsized 5CB layer was controlled by an electric field, a blue shift of band_1 was noticeable, up to $1.5 \mathrm{~nm}$, as is illustrated in Fig. 4a. In this case, a change of the location of the band_1 results directly in the increase of the value of the refractive index of LC which tends to $n_{\mathrm{e}}$ of $5 \mathrm{CB}$. In contrast, when the impact of the electric field on the LPFG with a thin layer was investigated, a red shift, up to $5 \mathrm{~nm}$, was obtained. In this case, the presence of the electric field could cause the fol- 
lowing changes in the LC layer: its refractive index (tends to $n_{\mathrm{e}}$ of $5 \mathrm{CB}$ ) and its thickness (as a reminder, the layer has a thickness in the nanometer range). Thus, the value and direction of the band 1 shift is a consequence of the changes of these parameters.

\section{Conclusion}

In conclusion, we have demonstrated a loss filter with an electrical tuning capability, based on a LPFG with the $\mathrm{LC}$ as its active material. For such a device, the observed electrical sensitivity mainly results from the parameters of the LC layer which can be changed by an external electric field. Two cases of a thick LC layer were investigated. First, when the layer is in the order of a few $\mu \mathrm{m}$ (LC cell is filled in entirely by $5 \mathrm{CB}$ ). For this configuration, the LPFGs exhibited slight shifts in their resonance attenuation bands towards the shorter wavelengths. In the second case, the nanosized LC layer formed on the bare LPFG significantly changed its spectral response. We observed a considerable red shift in the resonance wavelength. The LPFG with a nanosized LC layer showed one order of magnitude higher sensitivity than the LPFG with a microsized LC layer. Moreover, a "switching" effect, corresponding to the Fredericks transition in the $5 \mathrm{CB}$ LC, is much faster for the LPFG with a nanosized $5 \mathrm{CB}$ layer. The electrical field induces the wavelength shifts for the LPFGs with the micro- and nanosized LC layers which are plotted in Fig. 5. Their properties are

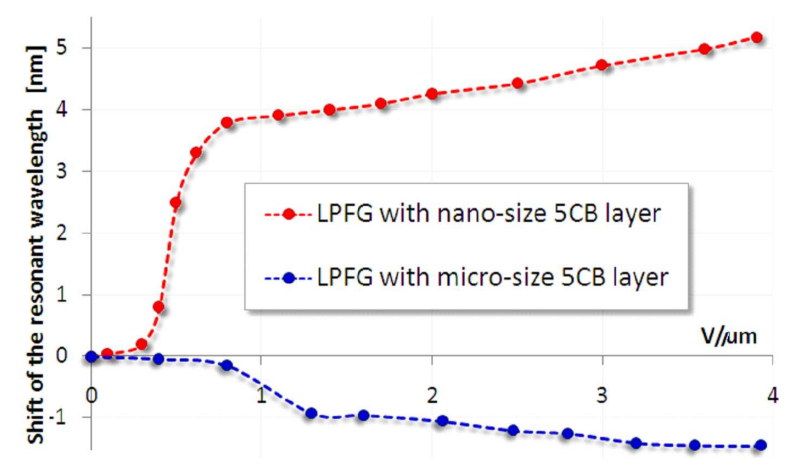

Fig. 5. comparison of the electrical sensitivity of the LPFG covered with a micro- and with a nanosized 5CB layer.

TABLE II

comparison LPFG with LC $5 \mathrm{CB}$ versus thickness of the 5CB layer for band_1.

\begin{tabular}{c|c|c}
\hline \hline Thickness of the 5CB layer & $\mu$ m scale & $\mathrm{nm}$ scale \\
\hline sensitivity to electric field $[\mathrm{nm} / \mathrm{V}]$ & 0.006 & 0.038 \\
electric field control range $[\mu \mathrm{m} / \mathrm{V}]$ & $0-1.5$ & $0-1$ \\
threshold electric field $[\mu \mathrm{m} / \mathrm{V}]$ & 0.8 & 0.2
\end{tabular}
compared in Table II. To conclude, the obtained experimental results have demonstrated that the presence of the LC as an active material enhances functionality of the LPFGs and could be applied toward further development of the practical tunable optical filters with a high electrical sensitivity.

\section{Acknowledgments}

This work was supported by the Natural Science and Engineering Research Council of Canada, by the Canada Research Chairs Programme.

The authors are also grateful to the Foundation for Polish Sciences (FNP) Professor's Subsidy "MISTRZ", to the European Union in the framework of the European Social Fund through the Warsaw University of Technology Development Program, and to the Fonds Nature et Technologies (FQRNT). The authors further thank P. Mikulic (PRC, Gatineau, Canada) for providing the long-period fiber gratings.

\section{References}

[1] O. Frazão, G. Rego, M. Lima, A. Teixeira, F.M. Araújo, P. André, J.F. Rocha, H.M. Salgado, in: Proc. London Communications Symp. 2001, Eds. Y. Beulachtar, G. Gavioli, University College of London, London 2001, p. 55.

[2] K. Seng Chiang, Q. Liu, in: Proc. ICOCN 2006, Ed. M. Syuhaimi, Chengu 2006, p. 128.

[3] S.W. James, R.P Tatam, Meas. Sci. Technol. 14, R49 (2003).

[4] D. Noordegraaf, L. Scolari, J. Legsgaard, L. Rindorf, T.T. Alkeskjold, Opt. Exp. 13, 7901 (2007).

[5] L.M. Tarakhan, Ukr. J. Phys. 51, 22 (2006).

[6] I. Gvozdovskyy, Yu. Kurioz, Yu. Reznikov, Opto-electron. Rev. 17, 116 (2009).

[7] L.M. Blinov, E.I. Kats, A.A. Sonin, Sov. Phys. Usp. 30, 604 (1987).

[8] R. Hou, Z. Ghassemlooy, A. Hassan, C. Lu, K.P. Dowke, Meas. Sci. Technol. 12, 1709 (2001).

[9] S.Z. Yin, K.W. Chung, X. Zhu, Opt. Commun. 188, 301 (2001).

[10] A. Czapla, W.J. Bock, T.R. Wolinski, P. Mikulic, in: 21st Canadian Conf. on Electrical and Computer Engineering, IEEE, Canada 2008, p. 117.

[11] H. Luo, X. Li, S. Li, J. Chen, Appl. Opt. 48, F95 (2009).

[12] I. DelVillar, I.R. Matias, F.J. Arregui, IEEE Photon. Technol. Lett. 17, 1893 (2005).

[13] A. Cusano, A. Iadicicco, P. Pilla, L. Contessa, S. Campopiano, A. Cutolo, Opt. Exp. 14, 19 (2006).

[14] T. Erdogan, J. Opt. Soc. Am. A 14, 1760 (1997).

[15] J.G. Delly, on-line J. Mod. Microsc. 107, 14255 (2003).

[16] D. Budaszewski, A. Domanski, Acta Phys. Pol. A 116, 285 (2009).

[17] A. Domanski, P. Lesiak, K. Milenko, D. Budaszewski, M. Chychlowski, S. Ertman, M. Tefalska, T.R. Wolinski, K. Jedrzejewski, L. Lewandowski, W. Jasiewicz, J. Helsztynski, A. Boczkowska, Acta Phys. Pol. A 116, 294 (2009).

[18] X. Shu, X. Zhu, S. Jiang, W. Shi, D. Huang, Electron. Lett. 35, 1580 (1999). 